\title{
Manejo estético y endodóncico de dientes con formación radicular incompleta
}

\section{Aesthetic and endodontic management of teeth with incomplete root formation}

\author{
Dávila Rodríguez LA*, Barcha Barreto DA**, León Barrios E***, \\ Simancas Pallares $M A^{* * * * *}$
}

\section{RESUMEN}

La necrosis pulpar es una de las principales complicaciones de los traumatismos dentales lo que implica en un diente inmaduro la interrupción de la maduración de la raíz y el cierre apical. Una terapia llamada "apexificación" se requiere para inducir la formación de una barrera calcificada apical permitiendo un relleno permanente y hermético de la raíz. Numerosos materiales han sido recomendados para inducir apexificación y conservar la estética en dientes con ápices inmaduros como el mineral trióxido agregado (MTA), el hidróxido de calcio [Ca $(\mathrm{OH}) 2]$, los postes de fibra de vidrio y entre los diversos materiales utilizados en prótesis, la cerámica que reproduce la apariencia de la dentición natural y tiene un comportamiento óptico muy similar a la de los tejidos duros dentales. El presente caso reporta paciente masculino de 17 años de edad con fractura coronaria complicada, conducto radicular expuesto a cavidad oral con formación radicular incompleta.

El objetivo del presente caso clínico es describir la apexificación como primera alternativa para mantener un diente útil, utilizando la formación de una barrera apical con mineral trióxido agregado (MTA) y recuperar la estética del paciente.

Conclusiones: La medicación intraconducto adecuada para la inducción a la formación de un cierre apical en un diente inmaduro o con formación radicular incompleta, es de trascendental importancia para el éxito del proceso de apexificación en donde juega un papel importante el MTA ya que en la actualidad es el material que brinda más ventajas en esta práctica como inductor de dicho proceso.

Palabras clave: Apexificación, MTA, trauma dental, odontología cosmética, diente inmaduro.

\section{SUMMARY}

Pulpal necrosis is one of the main complications of dental trauma which implies an interruption of the root maturation and the apical closure of an immature tooth. Apexification is a therapy used for inducing the creation of an apical calcified barrier allowing a permanent filling of the root. Several materials have been recommended to induce apexification and preserve aesthetics in teeth with immature apex, such as mineral trioxide aggregate (MTA), calcium hydroxide [Ca $(\mathrm{OH}) 2]$, glass fiber posts, as well as the different materials used in prosthetic dentistry, ceramics that reproduce the appearance of the natural dentition and show an optic behavior very similar to the hard tissues of the teeth. This case reports a 17 year old male patient with a complicated crown fracture, exposed root canal in the oral cavity with incomplete root formation. The purpose of this clinical case is to describe the use of apexification as the first choice to keep a useful tooth, using the formation of an apical barrier with mineral trioxide aggregate (MTA) and recover the patient aesthetics.

* Odontóloga. Universidad del Sinú Elías Bechara Zainúm.

** Odontólogo. Especialista en Endodoncia. Docente Universidad del Sinú Elías Bechara Zainúm.

*** Odontólogo. Universidad de Cartagena.

**** Odontólogo. Universidad de Cartagena. M.Sc (C). Epidemiología Clínica. Universidad Nacional de Colombia. Coordinador de investigación Escuela de Odontología Universidad del Sinú. 
Conclusions: The appropriate intracanal medication for inducing the formation of an apical closure in an immature tooth or with an incomplete root formation, is of vital importance for the success of the process of apexification where MTA plays an important role due to the fact that right now is the material that gives more advantages in this particular practice as an inducer in that process.

Key words: Apexification, MTA, dental trauma, cosmetic dentistry, immature teeth.

Fecha de recepción: 1 de octubre de 2012.

Aceptado para publicación: 5 de noviembre de 2012.

Dávila Rodríguez LA, Barcha Barreto DA, León Barrios E, Simancas Pallares MA. Manejo estético y endodóncico de dientes con formación radicular incompleta. Av. Odontoestomatol 2013; 29 (4): 201-206.

\section{INTRODUCCIÓN}

El trauma de los dientes en desarrollo es frecuente en pacientes entre las edades de 8 y 12 años. Una complicación grave de estos traumas es la necrosis de la pulpa, cuya prevalencia varía según el tipo de traumatismo (1). De acuerdo a la intensidad y la forma del trauma, estos traumatismos se clasifican en dos grupos básicos: los que no involucran la pulpa (fractura coronaria no complicada) y los que exponen la misma (fractura coronaria complicada) (2-4). La finalización del desarrollo radicular y el cierre del ápice se produce hasta 3 años después de la erupción de los dientes. El tratamiento de la lesión pulpar durante este período ofrece un gran reto para el clínico. Dependiendo de la vitalidad de la pulpa afectada, dos enfoques son posibles: apexogénesis o apexificación. Apexogénesis es "una terapia pulpar vital que se realiza para fomentar el desarrollo continuo y la formación fisiológica del extremo de la raíz". Por otro lado, apexificación se define como "un método para inducir una barrera calcificada en una raíz con el ápice abierto o con el desarrollo apical de una raíz incompleta en dientes con pulpa necrótica" (5). Históricamente, los clínicos realizan apexificación con hidróxido de calcio [Ca $(\mathrm{OH}) 2]$ a largo plazo, con tasas de éxito entre 79-96\% (6). Pero, a pesar de los éxitos conseguidos con el hidróxido de calcio, la técnica de apicoformación con este material presenta varias desventajas, como son: las múltiples citas necesarias en un largo periodo de tiempo, la colaboración del paciente, el resultado impredecible de formación de una barrera apical, problemas estéticos, gastos y, sobre todo, susceptibilidad a microfiltraciones coronarias y a fracturas de estos dientes debili- tados (7). Recientemente, diversos autores promueven la apexificación en un solo paso mediante el uso mineral trióxido agregado (MTA), con tasas de éxito entre $81-100 \%(8,9)$. El MTA es un polvo que contiene partículas hidrófilas, y se endurece en menos de cuatro horas tras el contacto con la humedad. Los componentes principales de MTA incluyen silicato tricálcico, aluminato tricálcico, óxido tricálcico y óxido de silicato (10). Este material cuenta con muchas ventajas como la aplicación en una sola cita, el sellado apical que favorece la regeneración ósea y menos porcentaje de fracturas, también cuenta con algunas desventajas, entre las que encontramos: dificultad de preparación del material, de manejo e introducción en el conducto radicular, sobre todo en los dientes largos y estrechos, la posibilidad de sobreobturación, el alto costo del producto y la producción de cambios excesivos de coloración a nivel coronal (11-13). Teniendo en cuenta esto, diversos materiales se emplean en un intento de reforzar esta zona y recuperar la estética del diente, incluyendo compuesto reforzado con resina de cemento de ionómero de vidrio, los postes, la raíz monobloque y las carillas de porcelana (14). En la actualidad, el poste de aleación y el núcleo es el método más ampliamente adoptado (15). Varios estudios clínicos han informado sobre la estética, biocompatibilidad, y la durabilidad de carillas de porcelana las cuales se han empleado con una tasa de éxito de 10 a 15 años, éxito que se atribuye a la relación íntima alcanzada entre la restauración y la estructura del diente a través del cemento sellador $(17,18)$.

El objetivo del presente caso clínico es describir la apexificación como primera alternativa para mante- 
ner un diente útil, utilizando la formación de una barrera apical con mineral trióxido agregado (MTA) y recuperar la estética del paciente.

\section{PRESENTACIÓN DEL CASO}

Paciente masculino de 17 años de edad acude a consulta odontológica, comenta que hace 5 años aproximadamente recibió trauma a nivel de órganos dentarios anterosuperiores. Actualmente no refiere sintomatología alguna, dentro de los antecedentes médicos no refiere ninguno de importancia para el caso. A la inspección clínica se observa fractura coronaria complicada que compromete tercio medio e incisal en incisivo central superior derecho, cambio de color, apertura que expone conducto radicular a la cavidad oral, ausencia de mal olor y movilidad, el sondaje periodontal se encuentra dentro de los parámetros normales; no se hallaron otras alteraciones patológicas (Fig. 1). Radiográficamente se observa apertura con acceso al espacio pulpar, formación radicular incompleta, paredes dentinarias delgadas, ápice abierto y tejidos perirradiculares discontinuos (Fig. 2).

Se diagnosticó tratamiento endodóncico previamente iniciado en órgano dentario 11 , según la clasificación clínica de patología pulpar y periapical basada en la propuesta de la Asociación Americana de Endodoncia (19). Se realiza consentimiento informado al paciente, explicando las ventajas, desventajas, riesgos y posibles complicaciones del tratamiento, en el cual el paciente acepta iniciar el tratamiento.

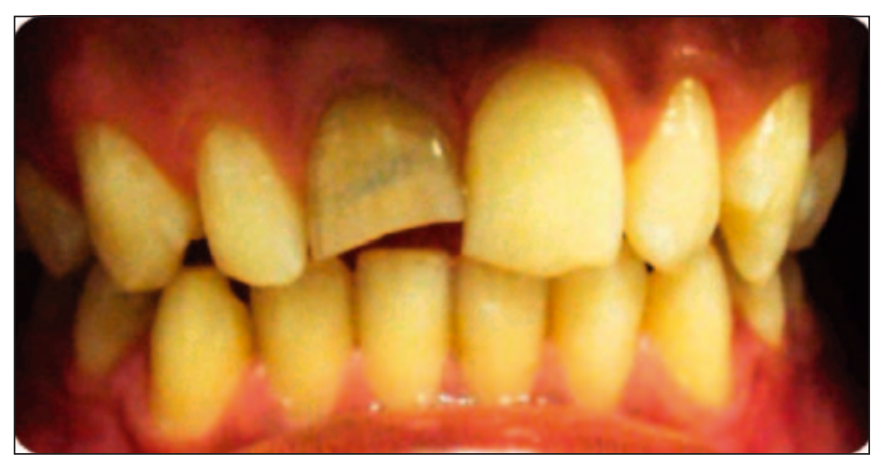

Fig. 1. Fractura coronal con destrucción del tercio medio e incisal en zona anterior en incisivo central inferior derecho.

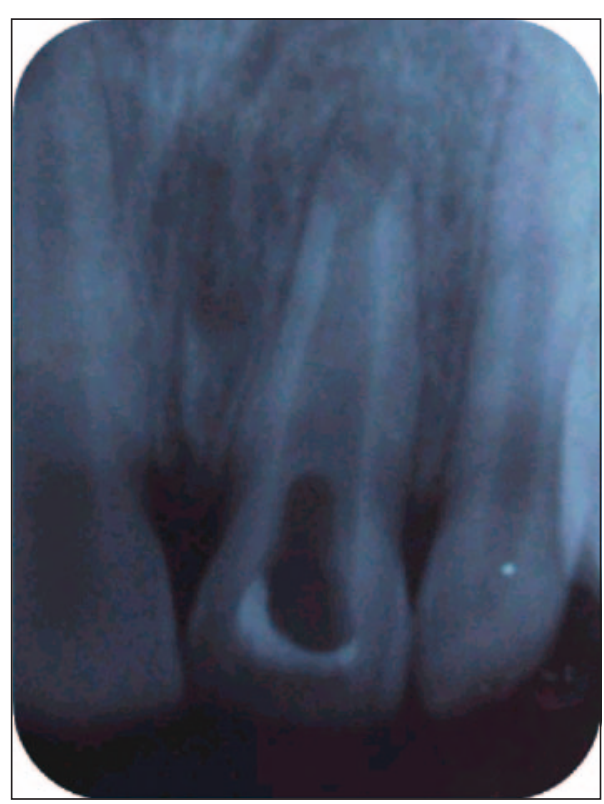

Fig. 2. Ilustra comunicación directa entre cámara pulpar y el medio intraoral, acceso al espacio pulpar y formación radicular incompleta (radiografía inicial).

Se decidió realizar apexificación con MTA (Fase 1). Cementación de poste adhesivo y carilla estética (Fase 2). El tratamiento se orientó a inducir la formación de una constricción apical de tejido duro. El procedimiento se realizó de la siguiente manera: El paciente se anestesió con técnica infiltrativa a nivel del surco vestibular, se realizó aislamiento con dique de goma, conductometría, adecuación del conducto para la obturación (limpieza, irrigación copiosa con hipoclorito de sodio, secado del conducto) obturación del conducto con MTA gris Angelus (Industria de productos odontológicos Ltda. Londrina PR - Brazil) (Fig. 3), cementación de poste adhesivo (Fig. 4), carilla estética (Fig. 5) y se realizó control radiográfico a los 2 meses donde se observa inducción del cierre apical (Fig. 6).

\section{DISCUSIÓN}

Cotarelo, C. et al (2005), afirmaron que el tratamiento con hidróxido de calcio hace que el pronóstico sea poco favorable, suponen que esto puede deberse al efecto proteolítico del hidróxido de calcio. Por el contrario promueven el empleo del MTA como tapón apical resaltando las propiedades de 


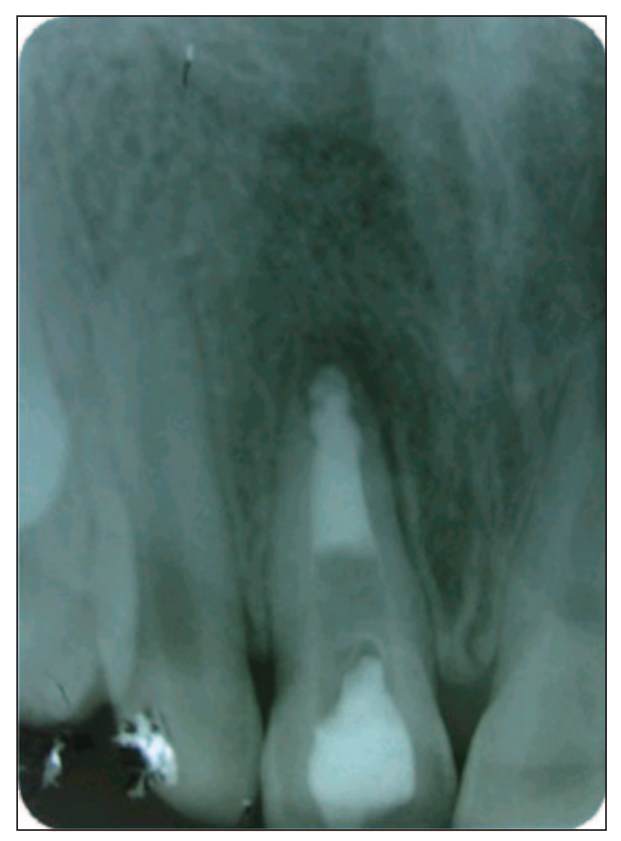

Fig. 3. Obturación con MTA a nivel del tercio apical y parte del tercio medio del conducto radicular.

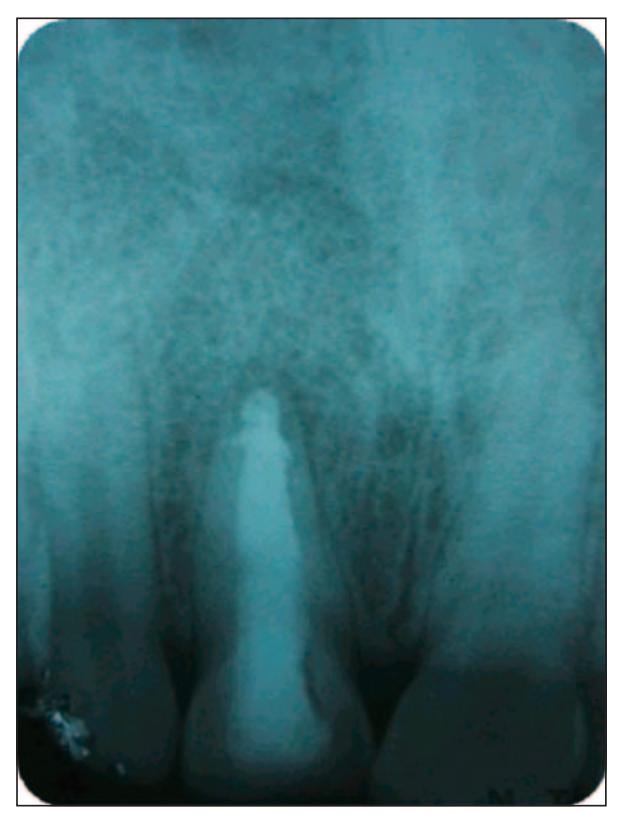

Fig. 4. Ilustra cementación de poste adhesivo y control a los 8 días de la obturación con MTA.

este material, las cuales hacen del MTA un material idóneo para tratamientos de apicoformación. También afirmaron que esta técnica permite reducir el tiempo de tratamiento necesario para una apicofor-

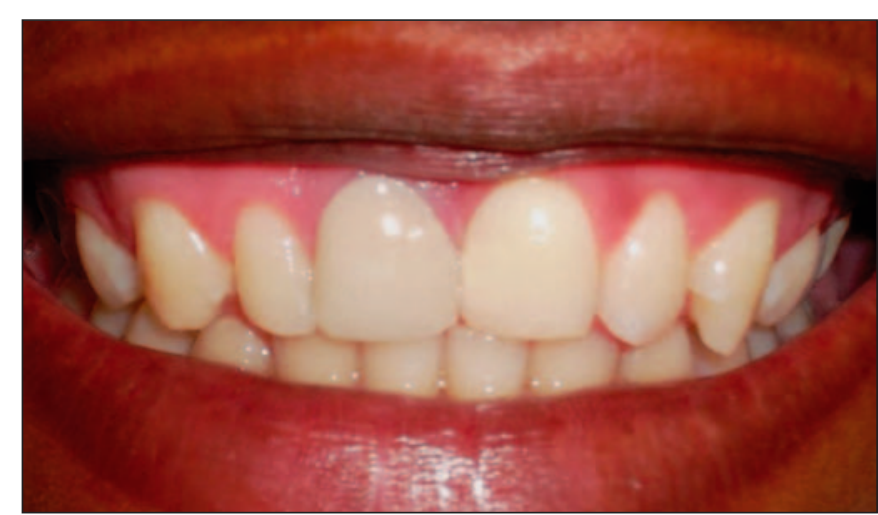

Fig. 5. llustra carilla estética en incisivo central superior derecho.

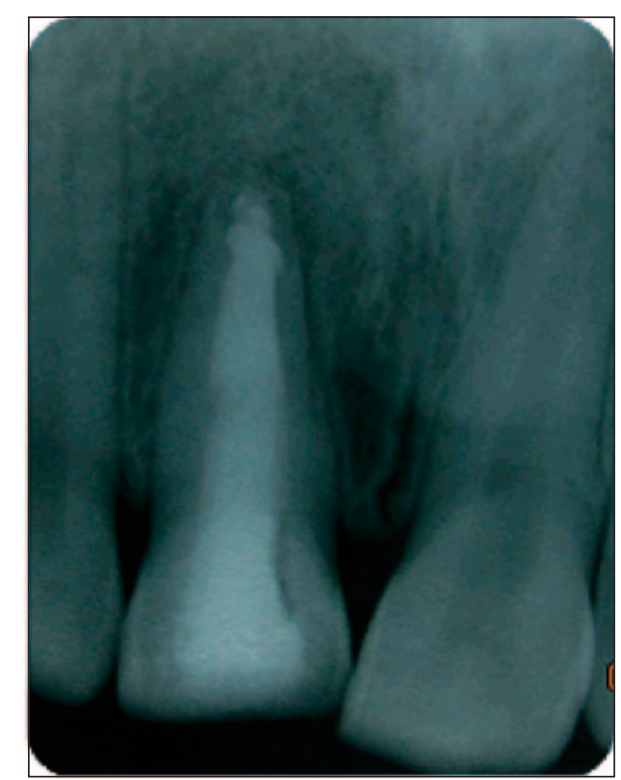

Fig. 6. Control radiográfico a los 2 meses donde se observa inducción del cierre apical.

mación clásica con hidróxido de calcio, lo cual coincidimos con el autor con respecto a lo descrito en el caso (20).

Las propiedades del MTA permiten hacer un refuerzo a las paredes debilitadas por la no vitalidad de la pulpa, aspecto en el que se está de acuerdo con Borras C, (2006) el cual afirmó que el procedimiento se complica cuando los dientes tienen paredes delgadas, para lo cual propone el relleno completo del canal con MTA, con la confianza de que este nuevo enfoque de tratamiento podría aumentar la resisten- 
cia del diente a la fractura y permitiría al paciente conservar la estética y la función por más tiempo (21). Para evitar este riesgo de fractura, varios autores proponen una técnica de apexificación en una visita, colocando un tapón apical de MTA en los últimos $5 \mathrm{~mm}$ del canal. Bortoluzzi et al., afirmó que los postes de fibra puestos significativamente refuerzan la zona cervical de los dientes y podrían disminuir el riesgo de fractura. Aspecto en el cual se está de acuerdo con el autor, ya que este hallazgo podría estar relacionado con el módulo de elasticidad similar entre el poste de fibra y la dentina distribuyendo equitativamente las fuerzas a lo largo de la raíz. Por ende, la obturación del conducto radicular y colocación de una restauración coronal en el diente inmediatamente son considerados como elementos clave para la conservación a largo plazo del diente tratado $(22,23)$.

\section{CONCLUSIÓN}

La medicación intraconducto adecuada para la inducción a la formación de un cierre apical en un diente inmaduro o con formación radicular incompleta, es de trascendental importancia para el éxito del proceso de apexificación en donde juega un papel importante el MTA ya que en la actualidad es el material que brinda más ventajas en esta práctica como inductor de dicho proceso.

\section{BIBLIOGRAFÍA}

1. Beslot A, Bonte E, Baune B, Serreau R, Aissat F, Quinquis L, et al. Mineral trioxyde aggregate versus calcium hydroxide in apexification of non vital immature teeth: Study protocol for a randomized controlled trial. BioMed Central, Trials 2011;12: 174.

2. Marcenes W, Tayfours Al, Issas S. Epidemiology of traumatic injuries to the permanent incisors of 9-12 years old School Children in Damasco, Siria. End Den Traumatol 1999;15:117-23.

3. Borsse E, Holm AK. Traumatic dental injuries in a cohort of 16 years old in Northern, Sweden. End Den Traumatol. 1997;13:276-8.
4. Glendor U, Koucheki B, Halling A. Risk evaluation and type of treatment of multiple dental trauma episodes to permanent teeth. End Den Traumatol 2000;16:205-10.

5. Moore A, Howley MF, O'Connell AC. Treatment of open apex teeth using two types of white mineral trioxide aggregate after initial dressing with calcium hydroxide in children. Dental Traumatology 2011; 27:166-73.

6. Kerekes K, Jacobsen I. Follow-up examination of endodontic treatment in traumatized juvenile incisors. Journal of Endodontics 1980; I Vol 6, NO 9.

7. Mente $\mathrm{J}$ et al. Mineral Trioxide Aggregate Apical Plugs in Teeth with Open Apical Foramina: A Retrospective Analysis of Treatment Outcome. JOE 2009; Vol 35.

8. Frank Al. Therapy for divergent pulpless tooth by continued apical formation. J Am Dent Assoc 1966;72:87-92.

9. Witherspoon DE, Small JC, Regan JD, Nunn $M$. Retrospective analysis of open apex teeth obturated with mineral trioxide aggregate. J Endod 2008;34:1171-6.

10. Schmoldt SJ, Kirkpatrick TC, Rutledge J, Yaccinoohn M. Reinforcement of Simulated Immature Roots Restored with Composite Resin, Mineral Trioxide Aggregate, Gutta-percha, or a Fiber Post after Thermocycling JOE, 2011; Vol 37.

11. Ghaziani P, Aghasiza NS, Nezami M. Endodontic treatment with MTA apical plugs: a case report. Journal of Oral Science 207;49(4):325-9.

12. Steinig $\mathrm{TH}$ et al. The use and predictable placement of mineral trioxide aggregate in onevisit apexification cases. Aust Endod J 2003;1: 34-42.

13. Torabinejad M, Chivian N. Clinical applications of mineral trioxide aggregate. J Endodon 1999;25: 197-205. 
14. Cristóbal B, Miñana M, Peix M. Mimaña. Apexificación con hidróxido de calcio vs. Tapon apical de MTA. Gaceta dental: Industria y profesiones 2005;159:58-79.

15. Catalan E, Sárries G, Giner L, Cortada M. Alternativa a las carillas de cerámica: las nuevas resinas compuestas. Dentum 2002;2(2):76-9.

16. Carossa S et al. Influence of Posts and Cores on Light Transmission Through Different All-Ceramic Crowns: Spectrophotometric and Clinical Evaluation. Int J Prosthodont 2001;14:9-14.

17. Altintas SH, Tak O, Secilmis A, Usumez A. Effect of Provisional Cements on Shear Bond Strength of Porcelain Laminate Veneers. European Journal of Dentistry. Eur J Dent 2011;5(4):373-9.

18. Türkaslan S, Utku U. Esthetic rehabilitation of crowded maxillary anterior teeth utilizing ceramic veneers: a case report. Cases Journal 2009;2: 8329.

19. AAE Consensus Conference Recommended Diagnostic Terminology. Journal of Endodontics 2009;35:(12)1634.

20. Borrás C, Estrela F. Utilización del MTA en el tratamiento de situaciones complejas de inmadu- rez radicular. Odontología Pediátrica 2006;14(3): 76-8.

21. Witherspoon DE, Ham K. One-visit apexification: technique for inducing root-end barrier formation in apical closures. Practical Periodontics and Aesthetic Dentistry 2001;13:455-60.

22. Andreasen JO, Munksgaard EC, Bakland LK. Comparison of fracture resistance in root canals of immature sheep teeth after filling with calcium hydroxide or MTA. Dental Traumatology 2006; 22:154-6.

23. Goldberg F, Kaplan A, Roitman M, Manfre S, Picca $M$. Reinforcing effect of a resin glass ionomer in the restoration of immature roots in vitro. Dental Traumatology 2002;18:70-2.

\section{CORRESPONDENCIA}

Miguel Ángel Simancas Pallares Universidad del Sinú Elías Bechara Zainúm Av. Pedro de Heredia, Sector María Auxiliadora Calle 30, no 39 - 175 Barrio Amberes

Colombia, Bolívar, Cartagena

E-mail: coord.inv.odontologia@unisinucartagena.edu.co 